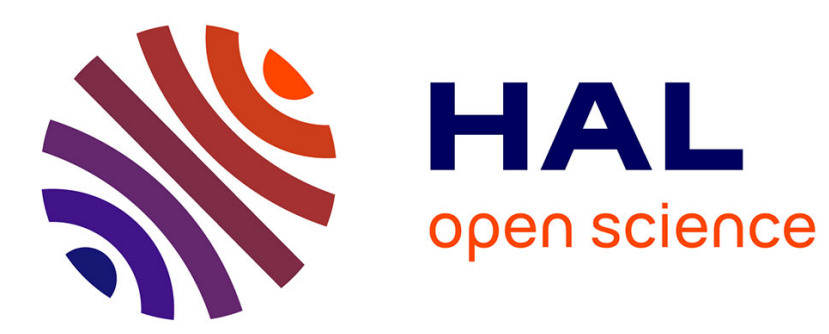

\title{
Modelling the pressure field in the vicinity of a microphone membrane using PIV
}

Olivier Richoux, Anne Degroot, Bruno Gazengel, Robert Macdonald, M. Campbell

\section{To cite this version:}

Olivier Richoux, Anne Degroot, Bruno Gazengel, Robert Macdonald, M. Campbell. Modelling the pressure field in the vicinity of a microphone membrane using PIV. 2009. hal-00400688

\section{HAL Id: hal-00400688 \\ https://hal.science/hal-00400688}

Preprint submitted on 1 Jul 2009

HAL is a multi-disciplinary open access archive for the deposit and dissemination of scientific research documents, whether they are published or not. The documents may come from teaching and research institutions in France or abroad, or from public or private research centers.
L'archive ouverte pluridisciplinaire HAL, est destinée au dépôt et à la diffusion de documents scientifiques de niveau recherche, publiés ou non, émanant des établissements d'enseignement et de recherche français ou étrangers, des laboratoires publics ou privés. 


\title{
Modelling the pressure field in the vicinity of a microphone membrane using PIV
}

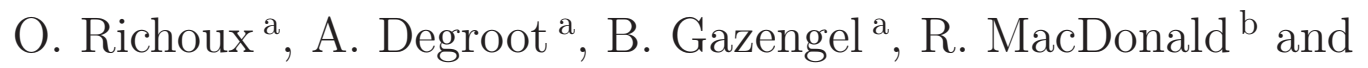 \\ M. Campbell ${ }^{\text {b }}$ \\ ${ }^{a}$ LAUM, CNRS, Université du Maine, Av. O. Messiaen, 72085 Le Mans, \\ FRANCE. \\ ${ }^{\mathrm{b}}$ Acoustics and Fluid dynamics Group, School of Physics, University of Edinburgh \\ Kings Buildings, Edinburgh, UK
}

\begin{abstract}
A new approach for for estimating the acoustic pressure in the near field of a microphone based on non-intrusive direct measurement of acoustic particle velocity is proposed.

This method enables the estimation of the acoustic pressure inside a domain located in front of the microphone membrane. The acoustic pressure is calculated using the acoustic particle velocity on the frontiers of this domain and a physical model based on the Green function of the system.

Results are obtained using the acoustic velocity measured with Particle Image Velocimetry (PIV) in front of a microphone excited with a plane wave inside a rectangular waveguide. They show that the diffraction of the plane wave by the microphone leads to an increase of the acoustic pressure on the microphone edge in the order of magnitude of $0.1 \mathrm{~dB}$.
\end{abstract}

Key words: PIV, pressure estimation, acoustic particle velocity measurement, integral formulation.

PACS: 43.20.Ye, 43.58.Kb, 43.58.Vb, 43.58.Fm

\section{Introduction}

Over the years, several methods have been developed for microphone calibration (for a review see [1]). These methods can be classified into two sorts : (i)

Email address: olivier.richoux@univ-lemans.fr (M. Campbell). 
relative calibration which provides an estimate of the sensitivity of a microphone as a function of a reference sensitivity and (ii) absolute calibration [2] which leads to an estimation of the sensitivity without any reference microphone.

For absolute calibration, the reciprocity technique is usually used which provides a typical precision of around $0.05 \mathrm{~dB}$ in an enclosed field configuration. In a free field configuration, this technique has been adapted [3,4] and standardized [5]. Nevertheless, free field absolute calibration suffers from numerous problems which are not resolved at present: the location of the acoustic center of the microphone is crucially important [6], the generation of standing waves between the different microphones perturbs the measurements and, generally, external reflections disturb the acoustic field in the vicinity of the microphone.

Microphone calibration using non-intrusive optical techniques such as Laser Doppler Velocimetry is proposed by some authors. These studies are mainly conducted in enclosed field conditions, more precisely in waveguides excited with plane waves. Two approaches are used.

On the one hand, authors consider that the impedance of the medium is known and measure the acoustic velocity at a single point. This supposes that the boundary conditions of the medium are perfectly known. We call it the "global approach". In this case, the acoustic pressure can be estimated at the measurement point thanks to the impedance. Acoustic pressure can also be estimated elsewhere using a propagation model of the system under study. Taylor [7], MacGillivray [8,9] and Koukoulas et al [10] use this approach. First results are encouraging: Taylor [7] shows that this method is accurate within $\pm 0.03 \mathrm{~dB}$ at $500 \mathrm{~Hz}$, Mac Gillivray et al [8,9] reach an accuracy of around $0.1 \mathrm{~dB}$ and Koukoulas et al [10] propose an accuracy of $0.2 \mathrm{~dB}$ at $170 \mathrm{~Hz}$.

On the other hand, authors consider a volume of fluid and measure the acoustic velocity on the volume boundaries. A propagating model of the fluid enables the estimation of the acoustic pressure everywhere in the volume. We call this the "local approach". For plane waves a slice of fluid is considered and the acoustic velocity measured at two points to estimate the acoustic pressure in the middle of the slice. Degroot et al [11] use this approach and show that using a $(u-u)$ probe with two LDV measurements provides a minimum uncertainty on the pressure estimation of $0.013 \mathrm{~dB}$ for frequencies of $1360 \mathrm{~Hz}$ and $680 \mathrm{~Hz}$.

The approaches described above for enclosed field calibration could be used in a free field. The first (global) approach only requires the measurement of the acoustic velocity at a single point but also requires that the impedance of the fluid for free field conditions is known. This can only be the case if the boundary conditions of the system are perfectly known, for example in a semi-infinite domain which can be reproduced with a semi anechoïc chamber. 
The second (local) approach does not require the boundary conditions of the system under study to be known but instead the acoustic velocity must be measured at a number of locations near the microphone membrane, in order to estimate the acoustic pressure on the membrane.

The local approach requires the characterization of the acoustic nearfield of a structure by LDV or other non-intrusive techniques. Previous work has been done by Gazengel et al [12], who measure the acoustic particle velocity in front of a loudspeaker to characterize its acoustic radiation in a free field using the LDV technique. Schedin et al [13] propose measuring the acoustic particle velocity in the vicinity of a plate using two-reference-beam doublepulsed holographic interferometry and Moreau et al characterize the acoustic field in a waveguide boundary layer using LDV and PIV techniques [14].

In this paper, we propose a preliminary study for calibrating microphones in free field conditions using a non-intrusive measurement technique and the local approach described above. Section two presents the general method used for estimating the acoustic pressure on the microphone membrane using the acoustic velocity measured at many locations near the membrane. Section three presents the experimental study of the acoustic field in the close vicinity of a microphone. In this section, the acoustic particle velocity is measured using a PIV technique. In the fourth section, a 2D model of the acoustic field near the microphone in derived using the Green function of the volume under study. Finally, the acoustic pressure field is estimated in front of the microphone membrane using the measured acoustic velocity and some discussion is given.

\section{General formulation of the acoustic pressure field in the vicinity of a microphone membrane}

In this section, a microphone excited with a plane wave is studied. An analytical development of the pressure field is proposed, considering a fluid domain (air) located in front of the microphone membrane (see Fig. 1). Acoustic pressure inside this volume can be estimated at the position $(r, \theta, z)=(\vec{w}, z)$ by means of measurement of normal velocity on the volume boundaries with an integral formulation.

The system under study (see Fig. 1 ) is an air fluid column with length $L$ and a circular section of radius $r_{a}$. The circular sections $\left(S_{1}, S_{2}\right)$ and the surface of the fluid column $S_{3}$ are subjected respectively to the normal acoustic velocities $v_{n_{1}}, v_{n_{2}}$ and $v_{n_{3}}$. As described in Fig. 1 and according to experimental results presented in section 3 , the normal acoustic velocities $v_{n_{1}}$ and $v_{n_{2}}$ are chosen to be uniform on the circular section and $v_{n_{3}}$ is considered to vary on the section 


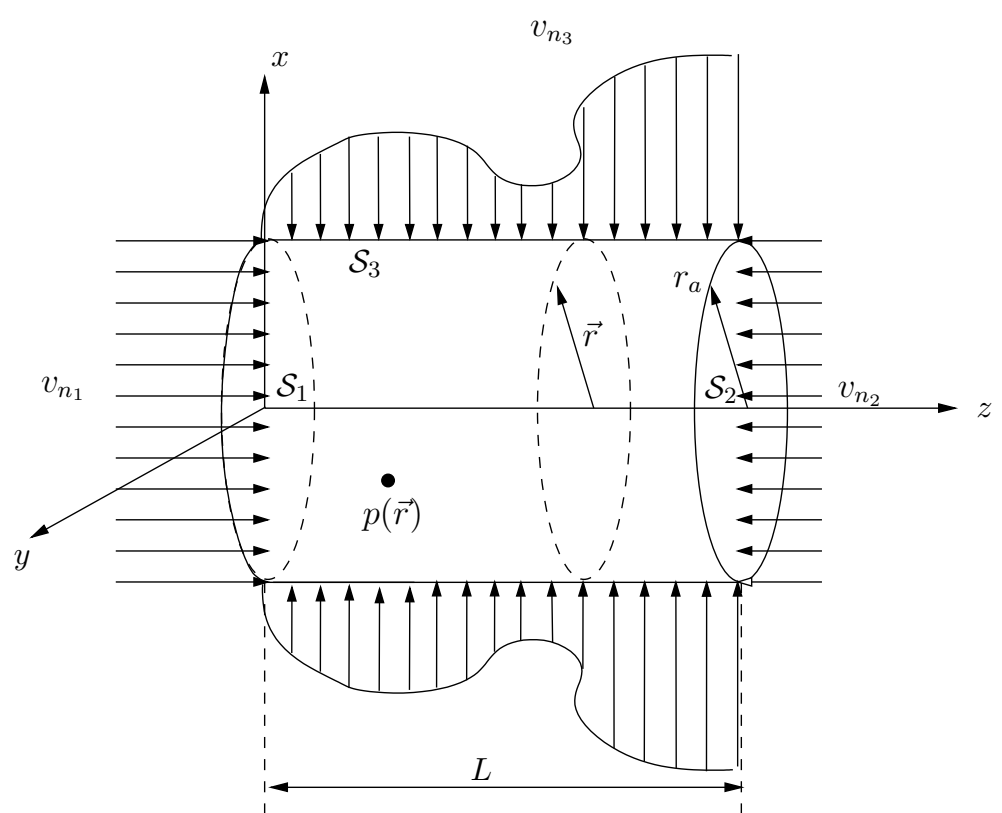

Fig. 1. Studied volume in front of the microphone.

A general integral formulation of the acoustic pressure in the volume $V$ is proposed hereafter.

In the frequency domain the acoustic pressure $p(\vec{r}, t)$ is written as

$$
p(\vec{r}, t)=p(\vec{r}) e^{j \omega t}
$$

where $\omega$ is the acoustic pulsation defined by $\omega=2 \pi f$. In the linear acoustic approximation and in the case of a perfect fluid, the acoustic propagation in the air fluid column $V$, is given by

$$
(\Delta+k 2) p(\vec{r})=0
$$

where $k=\omega / c_{0}$ is the wave number and $c_{0}$ is the sound celerity. The boundary conditions associated with eq. (2) are written

$$
\begin{aligned}
& \frac{\partial p(\vec{r})}{\partial n}=-j \omega \rho v_{n_{1}}(r) \text { for } r \in\left(0, r_{a}\right), \theta \in(0,2 \pi), z=0, \\
& \frac{\partial p(\vec{r})}{\partial n}=-j \omega \rho v_{n_{2}}(r) \text { for } r \in\left(0, r_{a}\right), \theta \in(0,2 \pi), z=L, \\
& \frac{\partial p(\vec{r})}{\partial n}=-j \omega \rho v_{n_{3}}(r) \text { for } r=r_{a}, \theta \in(0,2 \pi), z \in(0, L),
\end{aligned}
$$

where $\rho$ is the air density and $\partial / \partial n=\partial_{n}$ is the normal derivative to the surface $S$. The acoustic pressure field $p(\vec{r})$ in the volume $V$ (described by the 
closed surface $S$ ) is written, in this integral form, as []

$$
p(\vec{r})=\iiint_{V} G\left(\vec{r}, \vec{r}_{0}\right) f\left(\vec{r}_{0}\right) d V_{0}+\iint_{S}\left[G\left(\vec{r}, \vec{r}_{0}\right) \partial n_{0} p\left(\vec{r}_{0}\right)-p\left(\vec{r}_{0}\right) \partial n_{0} G\left(\vec{r}, \vec{r}_{0}\right)\right] d S_{0},
$$

where the function $f\left(\vec{r}_{0}\right)$ describes the sources distributed inside the volume $V$ and $G\left(\vec{r}, \vec{r}_{0}\right)$ is the Green function defined by the follonwing equation

$$
(\Delta+k 2) G\left(\vec{r}, \vec{r}_{0}\right)=-\delta\left(\vec{r}-\vec{r}_{0}\right) \text { in } V,
$$

and the boundary conditions on the surface $S$. In eq. $(7), \delta\left(\vec{r}-\vec{r}_{0}\right)$ is the Dirac distribution. The boundary conditions for the Green function are chosen as

$$
\begin{aligned}
& \partial_{n_{0}} G\left(\vec{r}, \vec{r}_{0}\right)=0 \text { for } r \in\left(0, r_{a}\right), \theta \in(0,2 \pi), z=0, \\
& \partial_{n_{0}} G\left(\vec{r}, \vec{r}_{0}\right)=0 \text { for } r \in\left(0, r_{a}\right), \theta \in(0,2 \pi), z=L, \\
& \partial_{n_{0}} G\left(\vec{r}, \vec{r}_{0}\right)=0 \text { for } r=r_{a}, \theta \in(0,2 \pi), z \in(0, L),
\end{aligned}
$$

to allow a description of the acoustic pressure field as a function of the acoustic velocity on the boundaries. Using eqs. (7), (8), (9) and (10), eq. (6), without any sources $\left(f\left(\vec{r}_{0}\right)=0\right)$, is written

$$
p(\vec{r})=\iint_{S} G\left(\vec{r}, \vec{r}_{0}\right) \partial n_{0} p\left(\vec{r}_{0}\right) d S_{0} .
$$

Eq. (11) combined with eqs. (3), (4) and (5) leads to the following expression for the acoustic pressure in the volume $V$

$$
p(\vec{r})=-j \omega \rho \iint_{S} G\left(\vec{r}, \vec{r}_{0}\right) v_{n_{0}} d S_{0}=-j \omega \rho \sum_{i} \iint_{S_{i}} G\left(\vec{r}, \vec{r}_{0}\right) v_{n_{i}} d S_{i},
$$

where $i=1,2$ and 3. Knowing the acoustic velocity on the surface $S_{1}, S_{2}$ and $S_{3}$ and the Green function of the system, the acoustic pressure can determined in the volume $V$ with the eq. (12).

\section{Experimental characterization of the acoustic velocity field in the vicinity of a microphone membrane}

In this section, the acoustic velocity field is estimated experimentally using Particle Image Velocimetry (PIV) inside the domain close to the microphone membrane as shown in figure 1 . The microphone is positioned inside a waveguide. PIV has previously been used to study acoustic flow in a waveguide [14]. The acoustic velocity field is measured inside a plane region (laser sheet) of dimensions $2 r_{a}, L$. 


\subsection{Experimental set-up}

\subsubsection{PIV system}

The PIV system uses a pulsed Cu-Laser Oxford Lasers $L S 20$ - 50 with a time between pulses of $20 \mu \mathrm{s}$. The mean power of the laser is $20 \mathrm{~W}$ and each pulse has a duration of $25-30 \mathrm{~ns}$ with a wavelength of $510.6 \mathrm{~nm}$ (green). The beam from the laser is converted to a light sheet for delivery to the region of interest. A CCD camera (Sensicam Double Shutter) is used for the acquisition of the PIV images, with a resolution $1280 \times 1024$ pixels. A Berkley Nucleonics model 500 A pulse generator is used in conjunction with National Instruments LabVIEW software to allow the capture of PIV image pairs at any phase in the period of the acoustic cycle. 30 measurements are made at a given phase and averaged to estimate the velocity field. Cross-correlation and post-processing are carried out on in-house PIV software.

\subsubsection{Acoustic system}

The experimental set-up is made up of a JBL $2446 \mathrm{H}$ loudspeaker mounted on a closed Perspex tube (with $10 \mathrm{~mm}$ wall thickness) with a length $L=0.5 \mathrm{~m}$ and a square section $S=0.1 \times 0.1 \mathrm{~m}^{2}$. The first cut-off frequency is $1720 \mathrm{~Hz}$ (first transverse mode). In the waveguide, a 1 inch $\mathrm{B} \& \mathrm{~K}$ microphone is placed parallel to the guide axis. Fig. (2) shows the acoustic set-up and the PIV system.

A stationary plane wave with a frequency $f=680 \mathrm{~Hz}$ is established in the waveguide. The microphone membrane is located between a node and an antinode of acoustic velocity to measure a sufficient acoustic velocity amplitude for PIV. The light sheet position is adjusted so as to graze the microphone membrane, in a plane corresponding to the diameter of the membrane. The waveguide is "seeded" using a SAFEX fog machine. The seeding is introduced during 3 to 5 seconds and 10 to 15 minutes are required before doing the measurement. The CCD Camera is positioned perpendicular to the light sheet and focused on the illuminated fog particles. The observation window corresponds to a rectangular section of the volume under study (dimensions $2 r_{a} \mathrm{x} L$ ). Measurements are realized for 20 equally spaced phase steps in the acoustic period (see Fig. 3). 


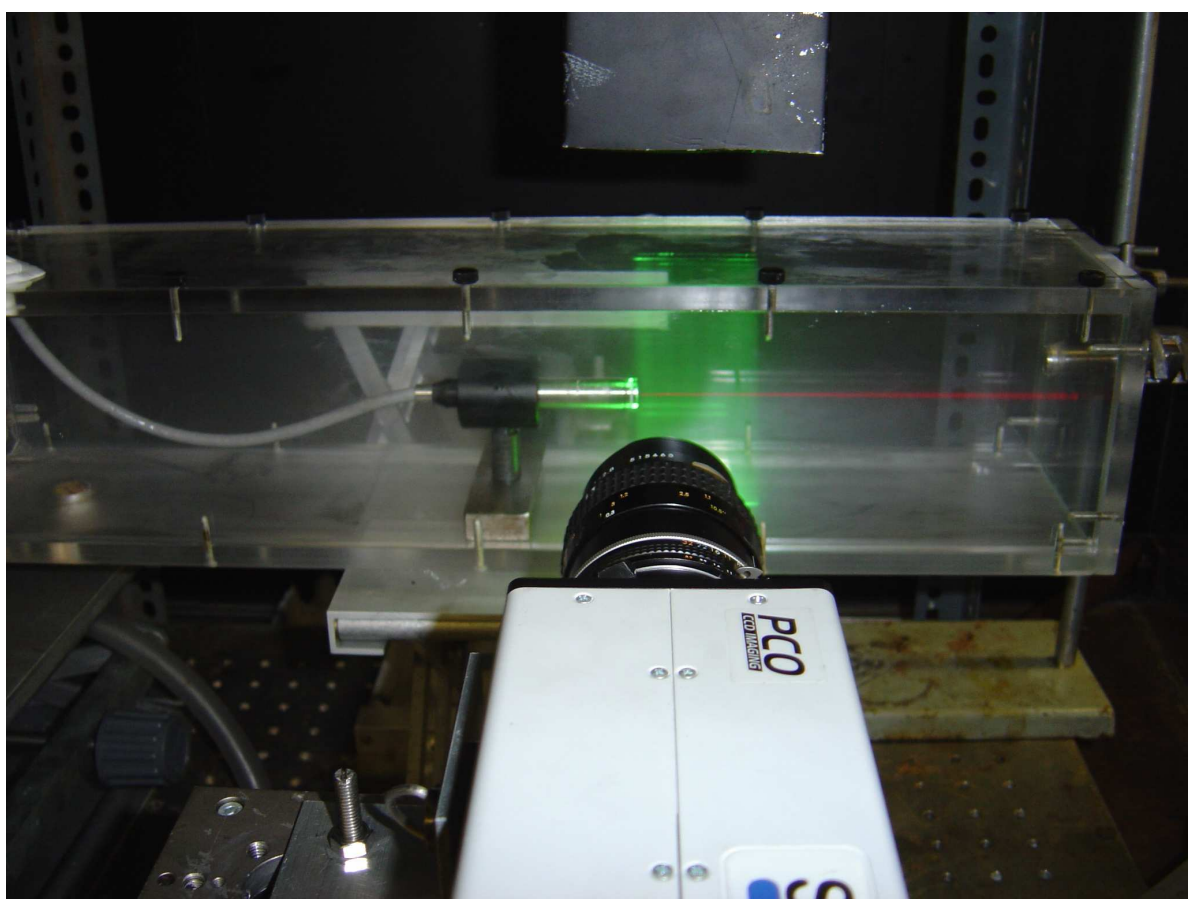

Fig. 2. Experimental set-up

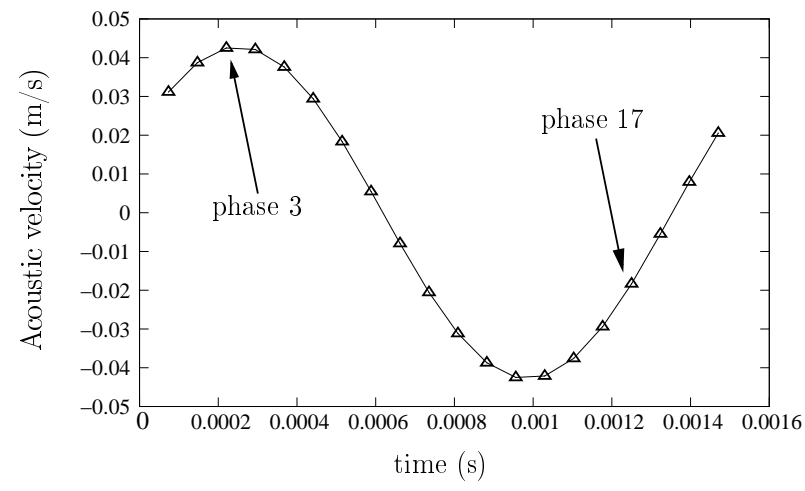

Fig. 3. View of the 20 measurement phases during one acoustic period.

\subsection{Experimental results}

\subsubsection{General analysis}

The acoustic velocity field measured for the phase 3 of the acoustic period is shown on the Fig. 4. The $z$-axis is the guide axis. The dimensions of the PIV image are $0.02 \times 0.024 \mathrm{~m}^{2}$. This PIV image highlights the evolution of the acoustic velocity field streamlines at the vicinity of the microphone membrane. In this representation, the velocity vectors go toward the membrane for a positive velocity amplitude. The shape of the field lines are approximately symmetrical around the microphone axis. 


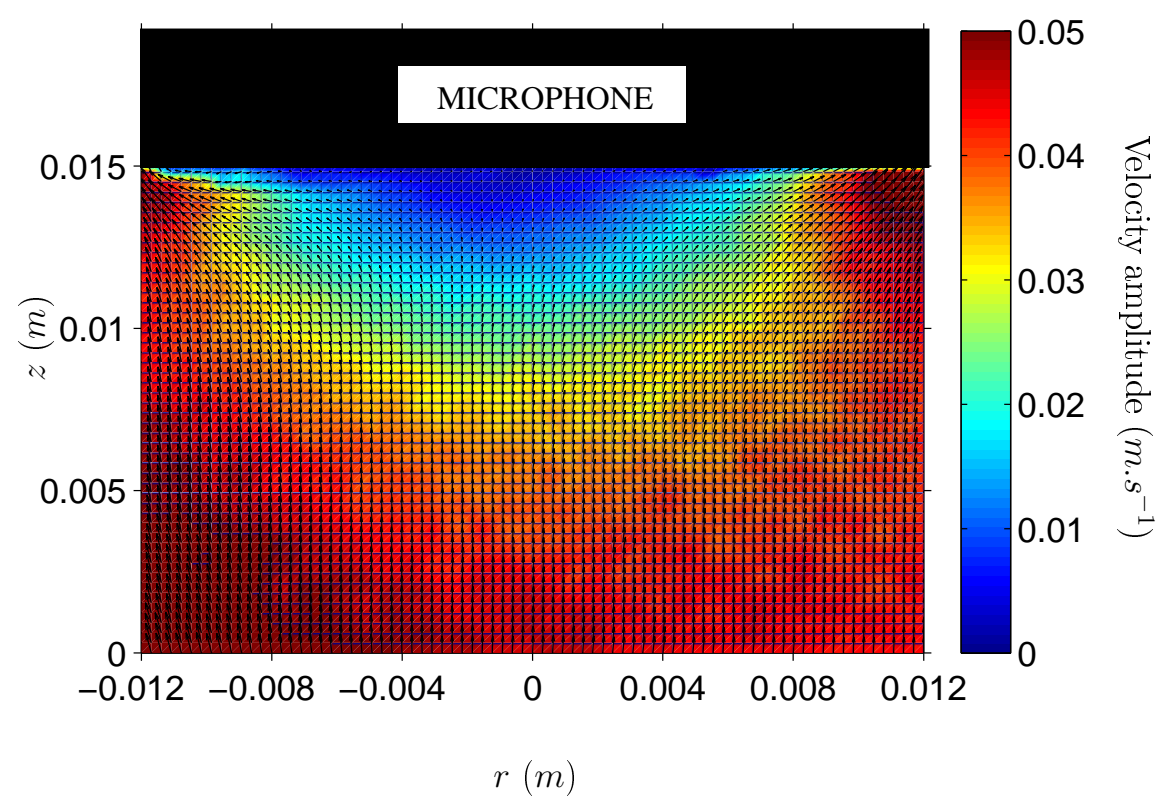

Fig. 4. View of experimental acoustic velocity field in the vicinity of the microphone for phase 3 .

\subsubsection{Longitudinal acoustic velocity}

Fig. 5 shows the longitudinal acoustic velocity $v_{z}$ as a function of $r$ for $z=0 \mathrm{~m}$ and for $z=0.015 \mathrm{~m}$. In this figure, the microphone membrane is located at $z=0.015 \mathrm{~m}$. This figure shows that the longitudinal velocity amplitude is almost constant for $z=0$. The acoustic wave can therefore be considered to be plane at this position (corresponding to surface $S_{1}$, see Fig. 1) in the domain under study. However, velocity $v_{n 1}$ shows a variation of $\pm 5.10^{-3} \mathrm{~m} . \mathrm{s}^{-1}$ around the mean value $\left(5.10^{-2} \mathrm{~m} . \mathrm{s}^{-1}\right)$, which introduces an uncertainty in the pressure estimation.

In the very closed vicinity of the microphone (the region defined by $r \in$ $[-0.004 ; 0.004] \mathrm{m}$ and $z \in[0.013 ; 0.015] \mathrm{m})$, the acoustic velocity field vanishes due to the membrane stiffness. For $r=0$, the amplitude of the acoustic velocity $v_{z}$ decreases from $0.045 \mathrm{~m} \cdot \mathrm{s}^{-1}(z=0 \mathrm{~m})$ to $2.10^{-3} \mathrm{~m} \cdot \mathrm{s}^{-1}(z=0.015 \mathrm{~m})$. Assuming the membrane velocity equals the acoustic velocity at $z=0.015 \mathrm{~m}$, this result shows that the membrane velocity is very small compared with the acoustic velocity (ratio $\simeq 1 / 22)$ measured at $z=0(15 \mathrm{~mm}$ from the membrane). This result should be confirmed by complementary direct measurements of the membrane velocity. In further works, the microphone membrane velocity should be measured by means of a Laser Vibrometer in order to confirm this hypothesis. 


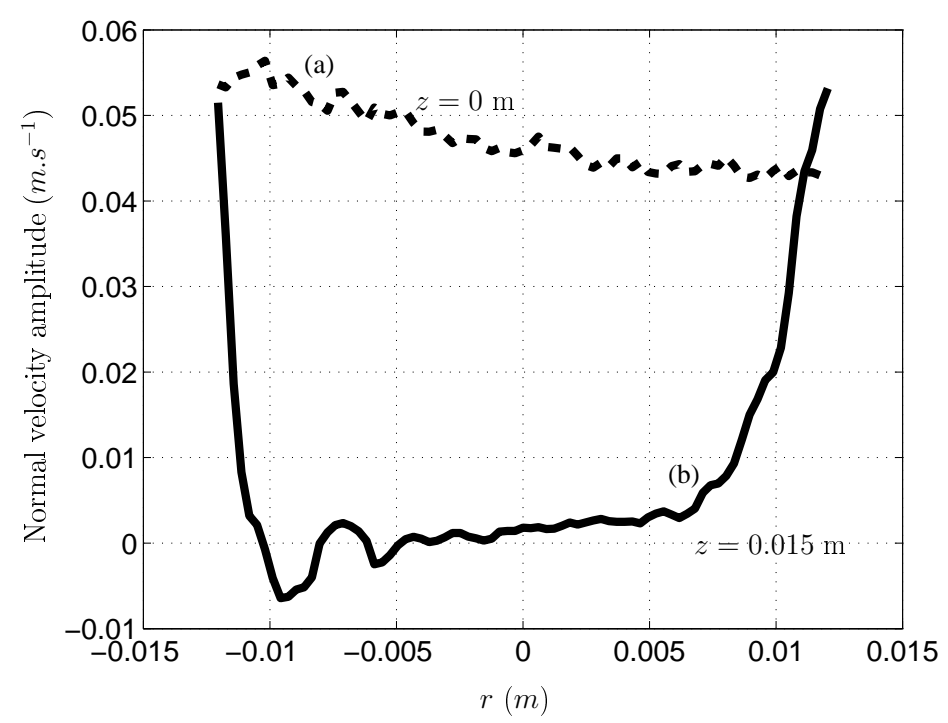

Fig. 5. Acoustic velocity amplitude $v_{z}$ as a function of $r$ for $z=0 \mathrm{~m}$ (a) and $z=0.015 \mathrm{~m}(\mathrm{~b})$.

\subsubsection{Radial acoustic velocity}

Figure $6 \mathrm{~A}$ shows the radial acoustic velocity amplitude $v_{r}$ as a function of $r$ for $z=0 \mathrm{~m}$ and $z=0.015 \mathrm{~m}$. In the very close vicinity of the membrane, the acoustic velocity amplitude $v_{r}$ increases with $r$. At $z=0.015 \mathrm{~m}$ and for $r=0 \mathrm{~m}$, the radial acoustic velocity amplitude is $v_{r}=1.10^{-4} \mathrm{~m} \cdot \mathrm{s}^{-1}$ and for $r=0.011 \mathrm{~m}, v_{r}=0.45 \mathrm{~m} \cdot \mathrm{s}^{-1} \cdot v_{r}$ is maximum for $r=0.012 \mathrm{~m}$ which illustrates the acoustic leakage at the edge of the microphone. In the vicinity of the microphone, the distribution of the radial velocity amplitude is not exactly symetric (Fig. 6A). This asymetry can be due to microphone misalignment, error in the velocity estimation with PIV or a weak air current caused by thermal effects. For greater distance from the microphone $(z<0.015 \mathrm{~m})$, the radial velocity amplitude becomes symetric as shown on Fig. $6 \mathrm{~B}$.

Finally, Fig. 7 illustrates the radial acoustic velocity amplitude $v_{r}$ as a function of $z$ for $r=0 \mathrm{~m}$ and $r=0.012 \mathrm{~m}$ corresponding to surface $S_{3}$ shown in Fig. 1. The radial acoustic velocity amplitude increases from zero $(r=0 \mathrm{~m})$ to $0.033 \mathrm{~m} . \mathrm{s}^{-1}(r=0.012 \mathrm{~m})$. This phenomenon illustrates again the presence of acoustic leakage on the edge of the microphone and has to be taken into account in equation 12 .

Taking these experimental results into account, we consider in the following that the longitudinal velocity $v_{n_{1}}$ is constant, that the membrane velocity $v_{n_{2}}$ can be neglected $\left(v_{n_{2}}=0\right)$ and that the radial velocity $v_{n_{3}}$ depends on the $z$ coordinate. The profile of $v_{n_{3}}$ as a function of $z$ is estimated analytically in the following $(\S 5)$. 

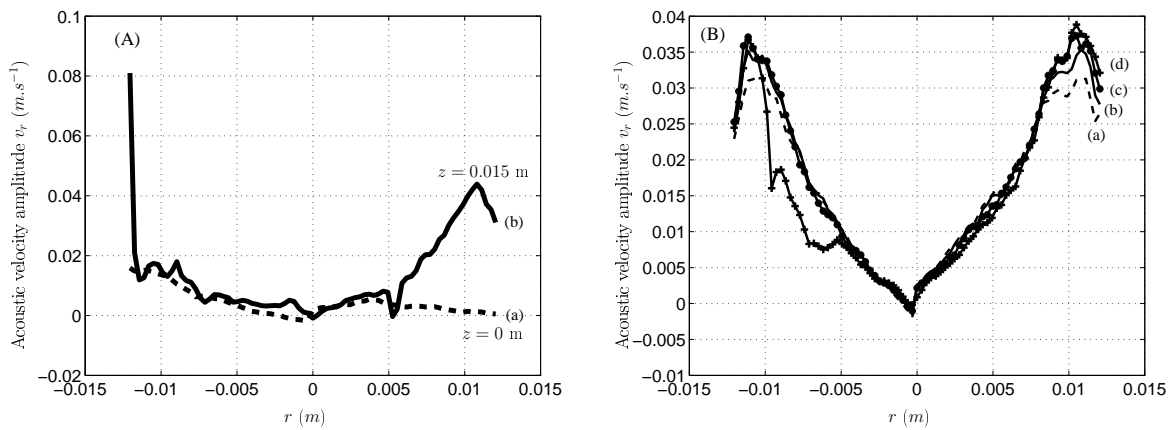

Fig. 6. (A) Radial acoustic velocity amplitude $v_{r}$ as a function of $r$ for $z=0 \mathrm{~m}$ (a) and $z=0.015$ (b). (B) Radial acoustic velocity amplitude $v_{r}$ as a function of $r$ for (a) $z=0.0136 \mathrm{~m} \mathrm{(..),} \mathrm{(b)} z=0.0139 \mathrm{~m} \mathrm{(--),} \mathrm{(c)} z=0.0142 \mathrm{~m}(*)$ and (d) $z=0.0145(+)$

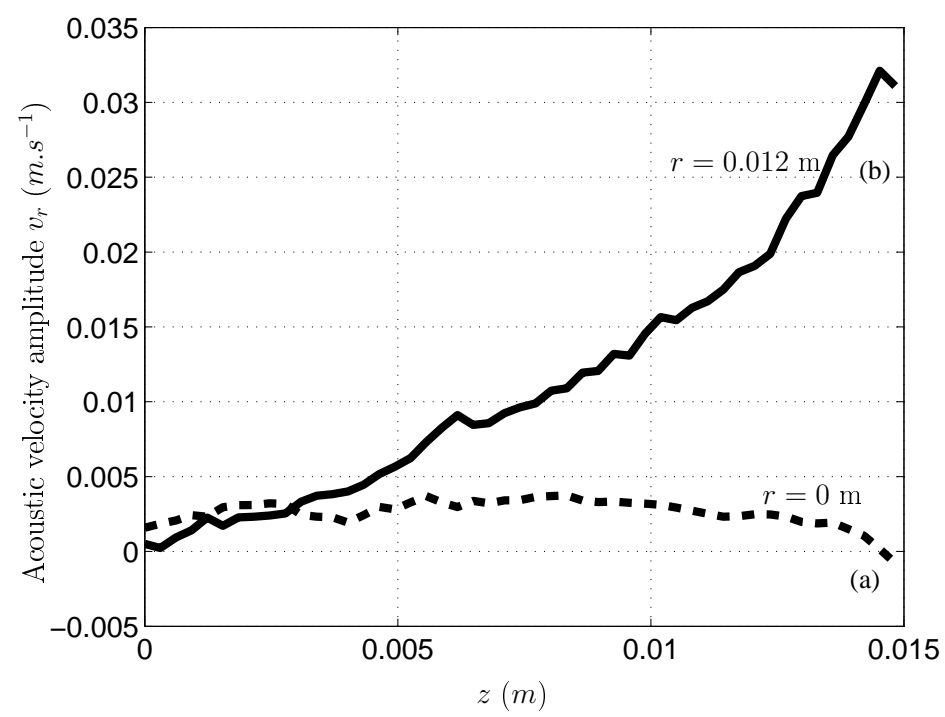

Fig. 7. Acoustic velocity amplitude $v_{r}$ as a function of $z$ for $r=0 \mathrm{~m}$ (a) and $r=0.012(\mathrm{~b})$.

4 Analytical model of the acoustic pressure field in the vicinity of a microphone membrane

In this section, we derive a specific expression of acoustic pressure in volume $V$ using the normal velocity profiles $v_{n_{1}}$ and $v_{n_{3}}$ estimated from experimental results (§3). We assume an axial symmetry of the system, which allows the derivation of a $2 \mathrm{D}$ model of the acoustic field. 


\subsection{Green function of the system}

The Green function $G\left(\vec{r}, \vec{r}_{0}\right)$ solution of the problem described by eq. (7), can be presented as

$$
\left(\Delta_{\vec{w}}+\delta_{z}+k 2\right) G\left(\vec{w}, z ; \vec{w}_{0}, z_{0}\right)=-\delta\left(\vec{w}-\vec{w}_{0}\right) \delta\left(z-z_{0}\right) \text { in } V,
$$

where $\Delta_{\vec{w}}=(1 / r) \partial_{r}\left(r \partial_{r}\right)+(1 / r 2) \partial_{\theta} 2$. The boundary conditions (eqs. (8), (9) and (10)) are expressed as following

$$
\begin{gathered}
\partial_{n_{0}} G\left(\vec{r}, \vec{r}_{0}\right)=\partial_{z_{0}} G\left(\vec{w}, z ; \vec{w}_{0}, z_{0}\right)=0 \text { for } z=0, \\
\partial_{n_{0}} G\left(\vec{r}, \vec{r}_{0}\right)=\partial_{z_{0}} G\left(\vec{w}, z ; \vec{w}_{0}, z_{0}\right)=0 \text { for } z=L, \\
\partial_{n_{0}} G\left(\vec{r}, \vec{r}_{0}\right)=\partial_{r_{0}} G\left(\vec{w}, z ; \vec{w}_{0}, z_{0}\right)=0 \text { for } r=r_{a} . .
\end{gathered}
$$

The Green function $G\left(\vec{w}, z ; \vec{w}_{0}, z_{0}\right)$ can be written as a discrete sum of eigenfunctions $\Psi_{\mu \nu}(\vec{w})$ of the cylinder $V$ under the form

$$
G\left(\vec{w}, z ; \vec{w}_{0}, z_{0}\right)=\sum_{\mu, \nu=0}^{\infty} g_{\mu \nu}\left(z, z_{0}\right) \Psi_{\mu \nu}\left(\vec{w}_{0}\right) \Psi_{\mu \nu}(\vec{w}),
$$

where $g_{\mu, \nu}\left(z, z_{0}\right)$ are dependent on the position $z$ and $\Psi_{\mu \nu}(\vec{w})$ are solutions of the following problem

$$
\begin{gathered}
\left(\Delta_{\vec{w}}+k_{w \mu \nu} 2\right) \Psi_{\mu \nu}(\vec{w})=-\delta\left(\vec{w}-\vec{w}_{0}\right) \forall r \in\left(0, r_{a}\right) \text { and } \forall \theta \in(0,2 \pi), \\
\partial_{n} \Psi_{\mu \nu}(\vec{w})=0 \text { for } r=r_{a} \text { and } \forall \theta \in(0,2 \pi) .
\end{gathered}
$$

The eigenfunctions $\Psi_{\mu \nu}(\vec{w})=\Psi_{\mu \nu}(r, \theta)$ take the following form

$$
\Psi_{\mu \nu}(r, \theta)=A_{\mu \nu} \cos (\mu \theta) J_{\mu}\left(k_{w \mu \nu} r\right),
$$

where $J_{\mu}$ is the Bessel function and the eigenvalues $k_{w \mu \nu}$ are given by

$$
k_{w \mu \nu}=\frac{\gamma_{\mu \nu}}{r_{a}}, \text { with } J_{\mu}^{\prime}\left(\gamma_{\mu \nu}\right)=0
$$

The coefficients $A_{\mu \nu}$ are found using the orthogonality of the eigenfunctions and are expressed as

$$
A_{\mu \nu}=\frac{2}{\left(1+\delta_{\mu 0}\right) \pi r_{a} 2\left(1-\frac{\mu 2}{\gamma_{\mu \nu} 2}\right) J 2_{\mu}\left(\gamma_{\mu \nu}\right)} .
$$

Using eqs. (13), (17) and (18), the coefficients $g_{\mu \nu}\left(z, z_{0}\right)$ are solutions of the following relation

$$
\left(\delta_{z} 2+k 2_{z \mu \nu}\right) g_{\mu \nu}\left(z, z_{0}\right)=\delta\left(z-z_{0}\right) \text { for } z \in(0, L)
$$


with $k 2_{z \mu \nu}=k 2-k_{w \mu \nu} 2$. The boundary conditions are given by

$$
\begin{aligned}
& \partial_{z} g_{\mu \nu}\left(z, z_{0}\right)=0 \text { for } z=0, \\
& \partial_{z} g_{\mu \nu}\left(z, z_{0}\right)=0 \text { for } z=L,
\end{aligned}
$$

and the solution is written

$$
\begin{aligned}
& g_{\mu \nu}\left(z, z_{0}\right)=-\frac{\cos \left(k_{z \mu \nu} z\right) \cos \left[k_{z \mu \nu}\left(z_{0}-L\right)\right]}{k_{z \mu \nu} \sin \left(k_{z \mu \nu} L\right)} \text { if } z<z_{0}, \\
& g_{\mu \nu}\left(z, z_{0}\right)=-\frac{\cos \left(k_{z \mu \nu} z_{0}\right) \cos \left[k_{z \mu \nu}(z-L)\right]}{k_{z \mu \nu} \sin \left(k_{z \mu \nu} L\right)} \text { if } z>z_{0} .
\end{aligned}
$$

\subsection{General formulation of acoustic pressure in the volume $V$}

The acoustic pressure in the volume $V$ can now be determined by means of the Green function of the system and the normal acoustic velocity on the surface $S$. Using eq. (17) in eq. (12), the pressure is written as

$$
p(\vec{r})=-j \omega \rho \sum_{i} \iint_{S_{i}} \sum_{\mu, \nu=0}^{\infty} g_{\mu \nu}\left(z, z_{0}\right) \Psi_{\mu \nu}\left(\vec{w}_{0}\right) \Psi_{\mu \nu}(\vec{w}) v_{n_{i}} d S_{i},
$$

for $i=1,2$ and 3 corresponding respectively to the surface $S_{i}$ and the normal velocities $v_{n_{i}}$. Setting

$$
p_{i}(r, \theta, z)=-j \omega \rho \sum_{\mu, \nu=0}^{\infty} \iint_{S_{i}} g_{\mu \nu}\left(z, z_{0}\right) \Psi_{\mu \nu}\left(\vec{w}_{0}\right) \Psi_{\mu \nu}(\vec{w}) v_{n_{i}} d S_{i}
$$

the total pressure in the volume $V$ is written as

$$
p(r, \theta, z)=\sum_{i=1}^{3} p_{i}(r, \theta, z)
$$

where $i$ indicates the considered surface of the volume. The pressure due to the different surfaces can now be calculated separately to show the influence of each surface area.

\subsubsection{Calculation of the pressure field $p_{1}(r, \theta, z)$}

Using eqs. (20) and (21) in eq. (29), the pressure field $p_{1}(r, \theta, z)$ is expressed as 


$$
\begin{gathered}
p_{1}(r, \theta, z)=-j \omega \rho \sum_{\mu, \nu=0}^{\infty} g_{\mu \nu}(z, 0) A 2_{\mu \nu} \cos (\mu \theta) J_{\mu}\left(\frac{\gamma_{\mu \nu}}{r_{a}} r\right) 2 \pi \delta_{m 0} \\
\int_{0}^{r_{a}} v_{n_{1}}\left(r_{0}\right) J_{\mu}\left(\frac{\gamma_{\mu \nu}}{r_{a}} r_{0}\right) r_{0} d r_{0} d \theta_{0}
\end{gathered}
$$

where $v_{n_{1}}\left(r_{0}\right)$, the normal acoustic velocity field for $x=0$, is independant of $\theta$. Taking into account the cylindrical symmetry of the system, the acoustic pressure field $p_{1}$ is also independent of $\theta$ (which implies that $\mu=0$ ) and is written as

$$
p_{1}(r, z)=-j \omega \rho \sum_{\nu=0}^{\infty} g_{0 \nu}(z, 0) A 2_{0 \nu} J_{0}\left(\frac{\gamma_{0 \nu}}{r_{a}} r\right) 2 \pi \int_{0}^{r_{a}} v_{n_{1}}\left(r_{0}\right) J_{0}\left(\frac{\gamma_{0 \nu}}{r_{a}} r_{0}\right) r_{0} d r_{0} d \theta_{0}
$$

with

$$
g_{0 \nu}(z, 0)=-\frac{\cos \left(k_{z 0 \nu}(z-L)\right)}{k_{z 0 \nu} \sin \left(k_{z 0 \nu} L\right)} \text { for } z>0
$$

and

$$
A 2_{0 \nu}=-\frac{1}{r_{a} 2 \pi J_{0}\left(\gamma_{0 \nu}\right)}
$$

\subsubsection{Calculation of the pressure field $p_{2}(r, \theta, z)$}

The acoustic pressure field $p_{2}(r, \theta, z)$, considered as independent of $\theta$ (due to the cylindrical symmetry), can be expressed as

$$
p_{2}(r, z)=-j \omega \rho \sum_{\nu=0}^{\infty} g_{0 \nu}(z, L) A 2_{0 \nu} J_{0}\left(\frac{\gamma_{0 \nu}}{r_{a}} r\right) 2 \pi \int_{0}^{r_{a}} v_{n_{2}}\left(r_{0}\right) J_{0}\left(\frac{\gamma_{0 \nu}}{r_{a}} r_{0}\right) r_{0} d r_{0} d \theta_{0}
$$

with

$$
g_{0 \nu}(z, L)=-\frac{\cos \left(k_{z 0 \nu} z\right)}{k_{z 0 \nu} \sin \left(k_{z 0 \nu} L\right)} \text { for } z<L,
$$

and $A 2_{0 \nu}$ defined by eq. (34).

\subsubsection{Calculation of the pressure field $p_{3}(r, \theta, z)$}

The normal acoustic velocity $v_{n_{3}}$ on the surface $S_{3}$, considered as independent of $r$ and $\theta$ (due to the cylindrical symmetry), depends only on the $z$ coordinate. The acoustic pressure field $p_{3}(r, \theta, z)$, considered as independent of $\theta$ (due to the cylindrical symmetry), is expressed as a function of the normal acoustic velocity $v_{n_{3}}(z)$ on the surface $S_{3}$ in using eqs. (20) and (21)

$$
p_{3}(r, z)=-j \omega \rho \sum_{\nu=0}^{\infty} A 2_{0 \nu} J_{0}\left(\gamma_{0 \nu}\right) J_{0}\left(\frac{\gamma_{0 \nu}}{r_{a}} r\right) 2 \pi r_{a} \int_{0}^{L} v_{n_{3}}\left(z_{0}\right) g_{0 \nu}\left(z, z_{0}\right) d z_{0} .
$$


The expression for $g_{0 \nu}\left(z, z_{0}\right)$ depends on the acoustic velocity profile $v_{n_{3}}(z)$ on the surface $S_{3}$.

\subsection{Calculation of acoustic pressure in the volume $V$}

Using eqs. (30), (32), (35) and (37) and, as shown by the experimental results (section 3.2), considering a incident plane wave on the surface $S_{1}$ and $v_{n_{2}}(r)=$ 0 on the surface $S_{2}$, the total pressure field in the volume $V$ can be written as

$$
\begin{gathered}
p(r, z)=j \omega \rho \frac{\cos [}{k(z-L)]} v_{n_{1}}+j \frac{2 \omega \rho}{r_{a}}\left(\sum_{\nu=0}^{\infty} \frac{J_{0}\left(\frac{\gamma_{0 \nu}}{r_{a}} r\right)}{J_{0}\left(\gamma_{0 \nu}\right)} \frac{1}{k_{z 0 \nu} \sin \left(k_{z 0 \nu} L\right)}\right. \\
\left\{\cos \left[k_{z 0 \nu}(z-L)\right] \int_{0}^{z} v_{n_{3}}\left(z_{0}\right) \cos \left[k_{z 0 \nu} z_{0}\right] d z_{0}\right. \\
\left.\left.+\cos \left[k_{z 0 \nu} z\right] \int_{z}^{L} v_{n_{3}}\left(z_{0}\right) \cos \left[k_{z 0 \nu}\left(z_{0}-L\right)\right] d z_{0}\right\}\right),
\end{gathered}
$$

where $A_{0 \nu} 2$ and $g_{0 \nu}\left(z, z_{0}\right)$ have been substituted by their expressions given by eqs. (34), (24) and (25).

To express the total pressure field in the volume $V$, the acoustic velocity profile $v_{n_{3}}(z)$ on the surface $S_{3}$ must be determined. The experimental results (section 3.2) suggest that this profile can be modeled by a parabolic curve defined as

$$
v_{n_{3}}(z)=-\eta z 2 \text { for } z \in[0, L] \text { and } \eta>0 .
$$

Finally, the calculation of the integral function in eq. (38) using eq. (39) leads to the following result for the total pressure in the volume $V$ (see appendix ??)

$$
\begin{aligned}
p(r, z)= & j \omega \rho \frac{\cos [k(z-L)]}{k \sin k L} v_{n_{1}}-j \frac{2 \omega \rho}{r_{a}}\left(\sum_{\nu=0}^{\infty} \frac{J_{0}\left(\frac{\gamma_{0 \nu}}{r_{a}} r\right)}{J_{0}\left(\gamma_{0 \nu}\right)} \frac{\eta}{k 2_{z 0 \nu} \sin \left(k_{z 0 \nu} L\right)}\right. \\
& \left.\times\left\{z 2 \sin \left(k_{z 0 \nu} L\right)+\frac{2}{k_{z 0 \nu}}\left[-\frac{1}{k_{z 0 \nu}} \sin \left(k_{z 0 \nu} L\right)+L \cos \left(k_{z 0 \nu} z\right)\right]\right\}\right)(40)
\end{aligned}
$$

where $k=k_{z 00}$.

Now, the characterization of the acoustic pressure field in the vicinity of the microphone (volume $V$ ) can be made with the knowledge of the normal acoustic velocities on the volume boundaries. 


\section{Results and discussion}

The calculation the acoustic pressure in front of the microphone requires the determination of the coefficient of the parabolic curve describing the variation of the normal acoustic velocity on the surface $S_{3}$. This coefficient $\eta$ defined in eq. (39) is determined by a minimization method. Fig. 8 shows a comparison between the experimental data and the model (defined by eq. (39)) with $\eta=$ $135 \mathrm{~m}^{-1} \cdot \mathrm{s}^{-1}$. PIV measurements of the acoustic velocity amplitude normal to $S_{3}$ have been obtained for the phase 3 of the acoustic period (see Fig. 3).

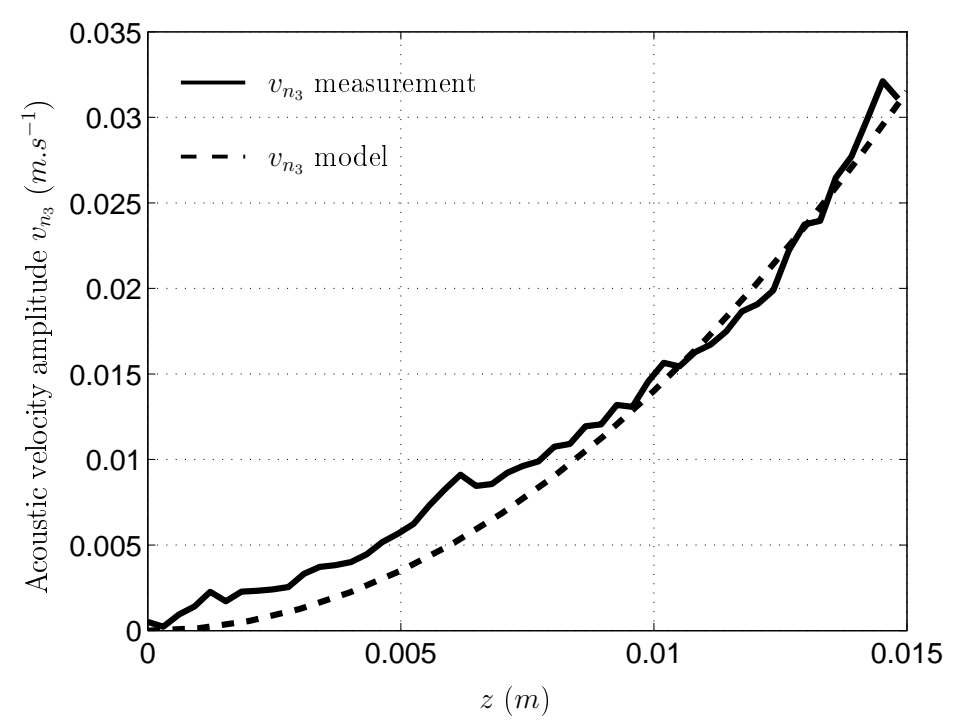

Fig. 8. Normal acoustic velocity amplitude on the surface $S_{3}$ as a function of $z$, experiment $(-)$ and model $(\cdots)$ defined by eq. (39).

Figs. 9 and 10 show the calculation of the pressure amplitude in the volume $V$ for $\nu=2$ modes and for $\nu=90$ modes respectively $(\nu>0$ corresponds to radial modes defined in eq. 40). The influence of the number of modes on the calculation of the acoustic pressure is clearly shown by comparing these two figures. The diffraction effects on the acoustic wave due to the microphone are visible even with 2 modes but this phenomenon is described more precisely when the modes number increases. According to the experimental results, the presence of the microphone distorts the velocity field streamlines allowing an acoustic leak on the border of the microphone (Fig. 4). This phenomenon leads to an increase of the acoustic pressure amplitude on the edge of the microphone. The acoustic pressure amplitude is not constant along the microphone membrane, varying from $126.65 \mathrm{~dB}$ at the microphone center to $126.8 \mathrm{~dB}$ at the microphone border. Due to the volume dimension and to the acoustic frequency $(f=680 \mathrm{~Hz}$ ), only the plane mode is propagative and all the higher modes are evanescent. The result of the calculation shows that the microphone edge creates diffraction of the plane wave which leads to the 
redistribution of the acoustic energy of all the modes and consequently to the excitation of higher modes.

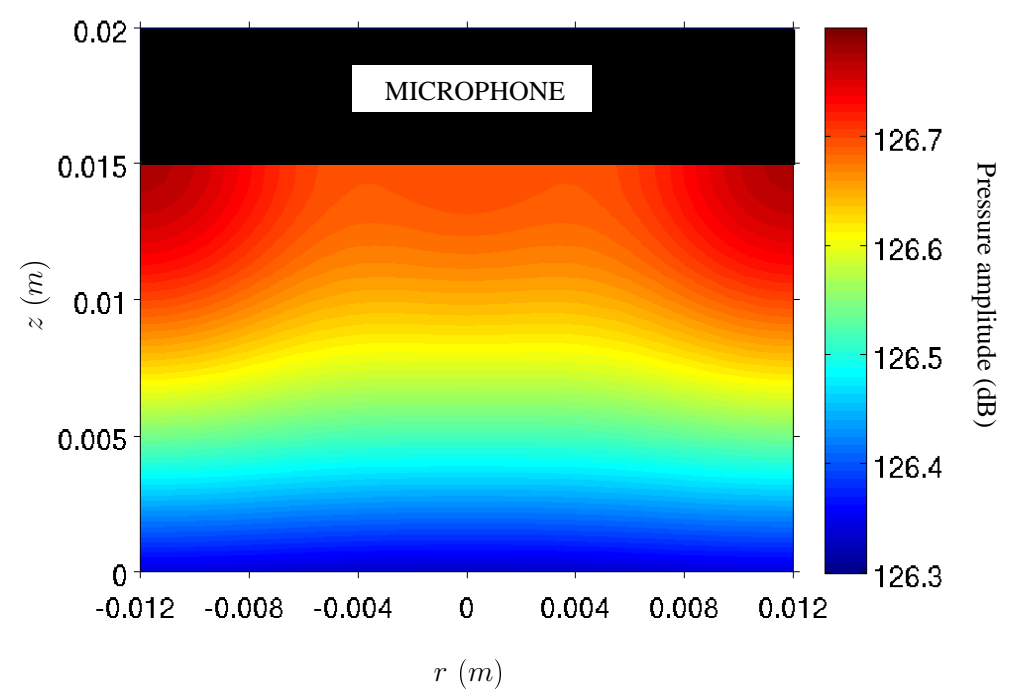

Fig. 9. Calculation of acoustic pressure amplitude in the vicinity of the microphone using eq. (40) with $\nu=2$.

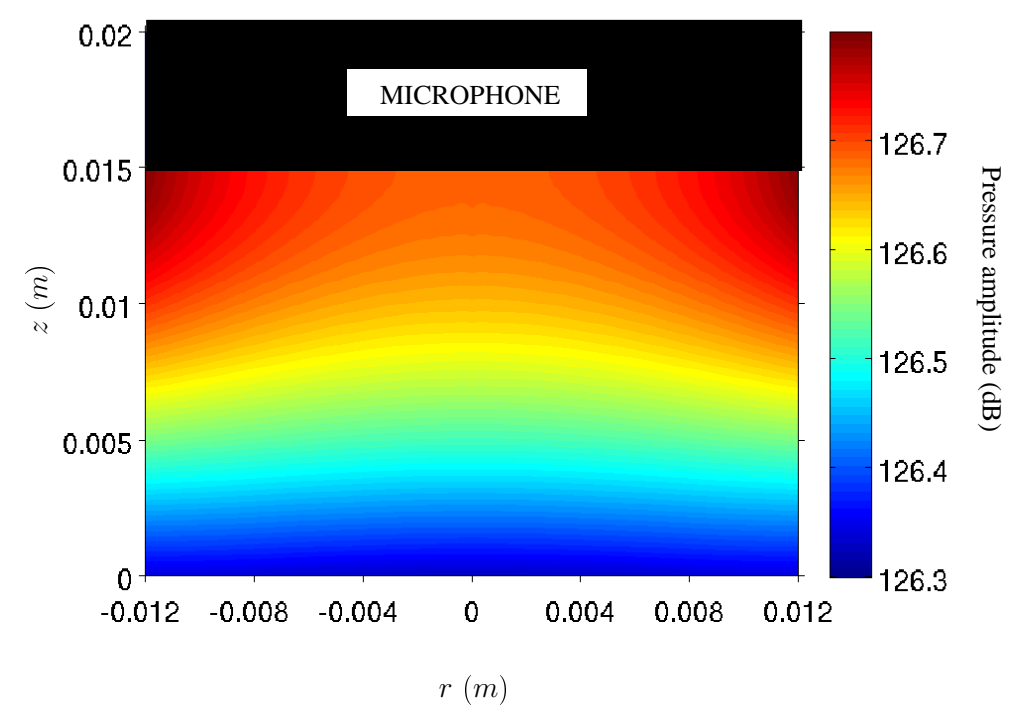

Fig. 10. Calculation of acoustic pressure amplitude in the vicinity of the microphone using eq. (40) with $\nu=90$.

\section{Conclusion}

A microphone subjected to a plane wave has been studied. Using a propagation model in a fluid domain located close to the microphone membrane and 
measuring the acoustic velocities on the boundary on the domain provides an estimate of the acoustic pressure on the microphone membrane. This model assumes that the normal acoustic velocity on the microphone membrane is uniformly zero and considers the incident wave as plane.

This preliminary study of the acoustic pressure field in the very close vicinity of a microphone highlights an acoustic pressure gradient along the microphone membrane in order of magnitude of $0.1 \mathrm{~dB}$. This acoustic pressure difference existing between the center and the edge of the membrane can be very problematic when microphone calibration requires an uncertainty less than $0.1 \mathrm{~dB}$. The hypothesis used in this work (incident plane wave, parabolic profile of radial velocity and motionless membrane) tend to minimize this pressure gradient value. A numerical calculation of integrals (Green fomulation) would enable to ignore these hypothesis and could lead to a more realistic pressure estimation.

This preliminary study opens new horizons in microphone calibration research, especially in free field conditions. These first results on the acoustic pressure field in the very close vicinity set an important question : what is measured by the microphone since the acoustic pressure is not uniform along the membrane ?

In the future, these results should be validated by measuring the acoustic pressure field in the very close vicinity of the microphone using, for instance, a small probe to not perturb the acoustic field. The proposed model can be improved by taking into account the membrane motion which could be estimated using laser probe measurements.

\section{References}

[1] AJ. Zuckenwar and GC. Herring Calibration of the pressure sensitivity of microphones by e free-field method at frequencies up to $80 \mathrm{kHz}$ J. Acoust. Soc. Am., 119(1):320-329, 2005.

[2] International Electrotechnical Commission IEC 61094-2:1992. Measurement microphone - Part 2: primary method for the pressure calibration of laboratory standard microphones by the reciprocity method. International Electrotechnical Commission; 1992.

[3] S. Barrera Figueroa, K. Rasmussen, F. Jacobsen. A time-selective technique for free-field reciprocity calibration of condenser microphones. J. Acoust. Soc. Am., 114(3):1467-76, 2003.

[4] S. Barrera Figueroa, K. Rasmussen, F. Jacobsen. On the interference between the two microphones in free-field reciprocity calibration. J. Acoust. Soc. Am., 116(5):2771-2778, 2004. 
[5] International Electrotechnical Commission IEC 61094-3:1995. Measurement microphone - Part 3: primary method for free field calibration of laboratory standard microphones by the reciprocity method. International Electrotechnical Commission; 1995.

[6] RP. wagner and V. Nedzelnitsky Determination of acoustic center correction values for type LS2aP microphones at normal incidence J. Acoust. Soc. Am., 104(1):192-1203, 1998.

[7] KJ. Taylor. Absolute calibration of microphone by a Laser-Doppler technique. J. Acoust. Soc. Am., 70(4):939-945, 1981.

[8] TJ. MacGillivray, DM. Campbell, CA. Greated, R. Barham. The development of a microphone calibration technique using Laser Doppler anemometry. Acustica-Acta Acustica, 88(1):135-41, 2002.

[9] TJ. MacGillivray, DM. Campbell, CA. Greated, R. Barham. The development of a microphone calibration technique using photon correlation spectroscopy. Acustica-Acta Acustica, 89(2):369-76, 2003.

[10] T. Koukoulas, P. Theobald, T. Schlicke and R. Barham. Towards a future primary method for microphone calibration: Optical measurement of acoustic velocity in low seeding conditions Optics and Lasers in Engineering, 46:791-796, 2008 .

[11] A. Degroot, R. MacDonald, O. Richoux, B. Gazengel and M. Campbell. Suitability of laser Doppler velocimetry for the calibration of pressure microphones Appl. Acoust., 69:1308-1317, 2008.

[12] B. Gazengel, O. Richoux and P. Rouquier. Characterization of a loudspeaker free field radiation by laser doppler velocimetry Acustica-Acta Acustica, 93(3):447-456, 2007.

[13] S. Schedin, A. O. Wahlin, and P. O. Gren. Transient acoustic near field in air generated by impacted plates J. Acoust. Soc. Am., 99(2):700-705, 1996.

[14] S. Moreau, R. Boucheron, H. Bailliet and J-C. Valire. Mesures LDV et PIV dans les couches limites acoustiques In $g^{i e m e}$ Congrès Francophone de Vélocimétrie Laser, Brussels, Belgium, 2004. 